\title{
An Efficient Estimation of the Mean Residual Life Function with Length-Biased Right-Censored Data
}

\author{
Hongping $\mathrm{Wu}^{1,2}$ and Yihui Luan ${ }^{1}$ \\ ${ }^{1}$ School of Mathematics, Shandong University, Jinan, Shandong 250100, China \\ ${ }^{2}$ College of Science, China University of Petroleum, Qingdao 266580, China \\ Correspondence should be addressed to Yihui Luan; yhluan@sdu.edu.cn
}

Received 20 March 2014; Accepted 26 May 2014; Published 9 June 2014

Academic Editor: Jian Guo Zhou

Copyright (C) $2014 \mathrm{H}$. Wu and Y. Luan. This is an open access article distributed under the Creative Commons Attribution License, which permits unrestricted use, distribution, and reproduction in any medium, provided the original work is properly cited.

\begin{abstract}
The mean residual life (MRL) function for a lifetime random variable $T^{0}$ is one of the basic parameters of interest in survival analysis. In this paper, we propose a new estimator of the MRL function with length-biased right-censored data and evaluate its performance through a small Monte Carlo simulation study. The results of the simulations show that the proposed estimator outperforms the existing one referred to in Data and Model Setup Section in terms of Monte Carlo bias and mean square error, especially when the censoring rate is heavy. We also show that the proposed estimator converges in distribution under some conditions.
\end{abstract}

\section{Introduction}

The MRL function at time $t$ is defined to be the expected remaining lifetime of a system given survival up to time $t$. It can be used to model various lifetime data in many areas of science. For example, a life insurance company may be interested in the life expectancy of a person, or an engineering firm may wish to estimate the expected remaining lifetime of a system, given survival up to time $t$. In literature, there has been a lot of work on the inference of the MLR function. Based on the i.i.d copies of the life time and the closed relationship between the MRL function and survival function, nonparametric estimators and NA based confidence intervals have been constructed (see $[1,2]$ ). For right-censored data, consistent estimator of the MRL function with its asymptotic normal distribution has been given by Yang [3] and Kumazawa [4]. EL approach has been developed to construct the confidence interval of the MRL function by Zhao and Qin [5], where, for censored data, the corresponding log-EL ratio was defined and the limiting distribution was proved to be a scaled $\chi^{2}$ distribution. A class of transformed MRL function for fitting survival data under right censoring was proposed and efficiency and robustness of estimators have been studied by Sun and Zhang [6]. A more recent work on estimation of the MRL with left-truncated and right-censored data has been constructed by Zhao et al. [7], and they showed that the proposed estimator converges weakly to a Gaussian process.

This paper considers survival data arising from lengthbiased sampling, which is a special case of left truncation. Following the terminology in the literature (see $[8,9]$ ), lengthbiased data are defined for left-truncated and right-censored data under the stationarity assumption, which means that the probability of the survival time being sampled is proportional to its length or the survival times are left-truncated by uniformly distributed random truncation times. Statistical inferences of length-biased data have been considered by many authors. Vardi [9] considered the nonparametric maximum likelihood estimator of the survival distribution. Gupta and Keating [10] obtained the relations for reliability measures of the length-biased distribution and some characterization results. Concerning the effect of the length bias on the sampling distribution of the covariates, Bergeron et al. [11] presented a joint likelihood approach and studied the large-sample behavior of the resulting maximum likelihood estimators. To estimate the survival distribution, Luo and Tsai [12] proposed a pseudopartial likelihood approach, and Huang and Qin [8] proposed a new nonparametric estimator based on the product-limit estimator. Their estimators were proved to be uniformly consistent and converge weakly to Gaussian processes. 
In this paper we propose a new estimator of the MRL function with length-biased and right-censored data. We compare the performance of our estimator with the existing one referred to in Section 2.1 through a small Monte Carlo simulation study and the results of the simulations show that the proposed estimator outperforms the existing one in terms of Monte Carlo bias and mean square error, especially when the censoring rate is heavy. The research is organized as follows. In Section 2, we introduce the model and the proposed estimator of the MRL function. The large sample property of our proposed estimator is stated in Section 3. The performance of the estimators is illustrated in Section 4.

\section{Notations and Estimators}

2.1. Data and Model Setup Section. Let $A^{0}$ and $T^{0}$ be two independent nonnegative random variables denoting the lefttruncated time and the lifetime of a system, respectively. Assume that the truncation probability $\alpha=\operatorname{Pr}\left(A^{0} \leq T^{0}\right)>$ 0 . Let $A$ and $T$ denote the observed truncation time and lifetime; that is, the pair $(A, T)$ is a realization from the conditional distribution of $\left(A^{0}, T^{0}\right)$ given that $A^{0} \leq T^{0}$. Under the stationarity assumption, $A$ is uniformly distributed on $[0, T]$, so $T$ has a length-biased distribution. Coupled with the censoring time $C$, which is applied to the residual lifetime $V=T-A$ instead of $T$ and independent of $(A, T)$, the observed data is composed of i.i.d. copies of $(A, Y, \delta)$, where $Y=\min (T, A+C), \delta=I(V \leq C)$. Now we suppose $\left\{\left(a_{i}, y_{i}, \delta_{i}\right)\right.$, $i=1,2, \ldots, n\}$ to be a sequence of i.i.d. random samples of $(A, Y, \delta)$ which one observes.

Denote by $f(t)$ and $S(t)$ the marginal density function and survival function of $T^{0}$ and by $g(t)$ and $G(t)$ the corresponding density function and survival function of the length-biased random variable $T$. Let $\tau=\inf \{t: S(t)=0\}$, $\tau \in R^{+} \cup\{+\infty\}$. Under length-biased sampling, $A$ and $T$ are stochastically dependent and $(A, T)$ has the joint density function (see [13])

$$
f_{A, T}(a, t)=\frac{f(t)}{\mu}, \quad 0 \leq a<t<\tau,
$$

and the density function $f$ of $T^{0}$ is related to the length-biased density function $g$ of $T$, as follows:

$$
g(t)=\frac{t f(t)}{\mu}, \quad 0 \leq t<\tau,
$$

where $\left.\mu=\left[\int_{0}^{\tau} u^{-1} g(u) d u\right)\right]^{-1}$ is finite.

According to the definition of the MRL function, the mean residual life of $T^{0}$ at time $0 \leq t<\tau$ is expressed as

$$
m(t)=E\left(T^{0}-t \mid T^{0}>t\right)=\frac{\int_{t}^{\tau} S(u) d u}{S(t)},
$$

and $m(t)=0$ when $t \geq \tau$.

In Huang and Qin [8], the nonparametric maximum likelihood estimator of $S(t)$ has been given:

$$
\widehat{S}(t)=\prod_{u \in[0, t]}\{1-d \widehat{\Lambda}(u)\},
$$

where $\widehat{\Lambda}$ is the estimated cumulative hazard function

$$
\widehat{\Lambda}(t)=\int_{0}^{t} \frac{d \bar{N}(u)}{\bar{R}(u)},
$$

with $\bar{N}(u)=n^{-1} \sum_{i=1}^{n} \delta_{i} I\left(y_{i} \leq u\right), \bar{R}(u)=n^{-1} \sum_{i=1}^{n} I\left(y_{i} \geq\right.$ $u)-\widehat{S}_{A}(u), \widehat{S}_{A}(u)=\prod_{v \in[0, u]}\{1-(d \widetilde{\mathrm{Q}}(v) / \widetilde{K}(v))\}, \widetilde{\mathrm{Q}}(v)=$ $n^{-1} \sum_{i=1}^{n} I\left\{I\left(a_{i} \leq v\right)+\delta_{i} I\left(y_{i}-a_{i} \leq v\right)\right\}$, and $\widetilde{K}(v)=$ $n^{-1} \sum_{i=1}^{n}\left\{I\left(a_{i} \geq v\right)+I\left(y_{i}-a_{i} \geq v\right)\right\}$.

Applying (3) and (4), when $0 \leq t \leq y_{(n)}$, the estimator of $m(t)$ can be naturally derived:

$$
\widehat{m}(t)=\frac{\int_{t}^{y_{(n)}} \widehat{S}(u) d u}{\widehat{S}(t)}
$$

where $y_{(n)}=\max \left(y_{1}, y_{2}, \ldots, y_{n}\right)$. If $t>y_{(n)}, \widehat{m}(t)=0$.

Let $S_{C}(\cdot)$ be the survival function of $C$ and $\tau_{C}=\inf \{t$ : $\left.S_{C}(t)=0\right\}$. Assume the following conditions hold:

(i) $L$ is a nonnegative constant satisfying $L<\sup \{t$ : $\left.S(t) S_{C}(t)>0\right\}$

(ii) $S$ and $S_{C}$ are absolutely continuous on $[0, L]$;

(iii) $\int_{t}^{\tau} g(u) d u<\infty, \int_{t}^{\tau} u^{-1} g(u) d u<\infty, t \in[0, \tau)$;

(iv) either $\tau_{C}<\tau$ with $S_{C}\left(\tau_{C}^{-}\right)>0$ or $\tau_{C} \geq \tau$.

As a straightforward result of the functional delta method, when $n \rightarrow \infty, \sqrt{n}(\widehat{m}(t)-m(t))$ converges in distribution to a zero mean normal variable with the variance function $\sigma^{2}(t)=S^{-4}(t)\left[S^{2}(t) E\left(\int_{t}^{\tau} Z(u) d u\right)^{2}-\right.$ $\left.2 S(t) \int_{t}^{\tau} Z(u) d u E Z(t) \int_{t}^{\tau} Z(u) d u+\left(\int_{t}^{\tau} S(u) d u\right)^{2} E Z^{2}(t)\right]$ for every $t \in[0, L]$ under Assumptions (i-iv).

In the next subsection, we shall state our proposed estimator of $m(t)$, and the simulation study in Section 4 shows that it performs better in several terms than the one in (6).

2.2. The Proposed Estimator. According to the joint density function of $(A, T)$, we can derive that

$$
\begin{aligned}
& E\left[\frac{I(Y \geq t) \delta}{S_{C}(Y-A)}\right] \\
& \quad=\int_{t}^{\tau} \frac{f(u)}{\mu} \int_{0}^{u}\left(\int_{u-a}^{\infty} f_{C}(v) d v\right) \frac{1}{S_{C}(u-a)} d a d u \\
& \quad=\int_{t}^{\tau} g(u) d u, \\
& E\left[\frac{I(Y \geq t) \delta}{Y S_{C}(Y-A)}\right] \\
& =\int_{t}^{\tau} \frac{f(u)}{\mu} \int_{0}^{u}\left(\int_{u-a}^{\infty} f_{C}(v) d v\right) \frac{1}{u} \frac{1}{S_{C}(u-a)} d a d u \\
& =\int_{t}^{\tau} u^{-1} g(u) d u,
\end{aligned}
$$

where $f_{C}(\cdot)$ is the density function of the censoring time $C$. 
Applying (7) we shall use the method of moments to derive an estimator for the MRL function $m(t)$. To proceed we note that $m(t)$ can be written as

$$
\begin{aligned}
m(t)=E\left[T^{0}-t \mid T^{0}>t\right] & =\frac{\int_{t}^{\tau} u f(u) d u}{\int_{t}^{\tau} f(u) d u}-t \\
& =\frac{\int_{t}^{\tau} g(u) d u}{\int_{t}^{\tau} u^{-1} g(u) d u}-t .
\end{aligned}
$$

An alternative estimator of the MLR function $m(t)$, for $0 \leq t \leq y_{(n)}$, can be derived as follows:

$$
\widetilde{m}(t)=\frac{(1 / n) \sum_{i=1}^{n} I\left(y_{i}>t\right) \delta_{i} / \widehat{S}_{C}\left(y_{i}-a_{i}\right)}{(1 / n) \sum_{i=1}^{n} y_{i}^{-1} I\left(y_{i}>t\right) \delta_{i} / \widehat{S}_{C}\left(y_{i}-a_{i}\right)}-t
$$

where $\widehat{S}_{C}(t)$ is the product-limit estimation of $S_{C}(t)$, and it is expressed as

$$
\widehat{S}_{C}(t)=\prod_{i=1}^{n}\left(1-\frac{\left(1-\delta_{i}\right) I\left(y_{i}-a_{i} \leq t\right)}{R\left(y_{i}-a_{i}\right)}\right),
$$

where $R(t)=\sum_{j=1}^{n} I\left(y_{j}-a_{j} \geq t\right)$. If $t>y_{(n)}, \widetilde{m}(t)=0$.

In the next section we shall establish that $\widetilde{m}(t)$ converges in distribution under some conditions by using the Hadamard derivative method.

\section{The Asymptotic Properties}

Firstly, let us introduce some notations and lemmas.

Denote

$$
\begin{gathered}
M(t)=\int_{t}^{\tau} g(u) d u \\
N(t)=\int_{t}^{\tau} u^{-1} g(u) d u, \\
\widehat{M}(t)=\frac{(1 / n) \sum_{i=1}^{n} I\left(y_{i}>t\right) \delta_{i}}{\widehat{S}_{C}\left(y_{i}-a_{i}\right)} \\
\widehat{N}(t)=\frac{(1 / n) \sum_{i=1}^{n} y_{i}^{-1} I\left(y_{i}>t\right) \delta_{i}}{\widehat{S}_{C}\left(y_{i}-a_{i}\right)} \\
\gamma(M(t), N(t))=m(t)=\frac{M(t)}{N(t)}-t, \\
\gamma(\widehat{M}(t), \widehat{N}(t))=\widetilde{m}(t)=\frac{\widehat{M}(t)}{\widehat{N}(t)}-t,
\end{gathered}
$$

and assume that

$$
\begin{gathered}
\iint \frac{1}{S_{C}(t-a)} F^{*}(d a, d t)<\infty, \\
\iint \frac{1}{t^{2} S_{C}(t-a)} F^{*}(d a, d t)<\infty,
\end{gathered}
$$

where $F^{*}(\cdot, \cdot)$ is the joint distribution function of $(A, T)$.

Lemma 1 gives the Hadamard derivative of $\gamma$ at $(M(t)$, $N(t))$.
Lemma 1. For $t \in[0, L]$, the function $\gamma$ is Hadamarddifferentiable at $(M(t), N(t))$, where its derivative is equal to

$$
\gamma_{(M(t), N(t))}^{\prime}\left(h_{1}(\cdot), h_{2}(\cdot)\right)=\frac{h_{1}(t)}{N(t)}-\frac{M(t) h_{2}(t)}{N^{2}(t)} .
$$

Lemma 2. Assume the conditions ( $i-i v)$ and (12) hold, for every $t \in[0, L]$, as $n \rightarrow \infty$. Then

(i) $Z_{1 n}(t) \hat{=} \sqrt{n}(\widehat{M}(t)-M(t))$ converges in distribution to a zero mean normal variable $Z_{1}$ with the variance function $E\left(I(Y \geq t) \delta / S_{C}^{2}(Y-A)\right)-M^{2}(t)$;

(ii) $Z_{2 n}(t) \hat{=} \sqrt{n}(\widehat{N}(t)-N(t))$ converges in distribution to a zero mean normal variable $Z_{2}$ with the variance function $E\left(I(Y>t) \delta / Y^{2} S_{C}^{2}(Y-A)\right)-N^{2}(t)$.

Proof. Firstly, let us give the proof of the result in Lemma 2(i). Under the regular conditions (i-iv) and (12), since $E(I(Y>$ $\left.t) \delta / S_{C}(Y-A)\right)=M(t)$, by the central limit theorem, we can derive

$$
\begin{aligned}
& \sqrt{n}\left(\frac{1}{n} \sum_{i=1}^{n} \frac{I\left(y_{i}>t\right) \delta_{i}}{S_{C}\left(y_{i}-a_{i}\right)}-M(t)\right) \\
& \stackrel{\mathscr{L}}{\longrightarrow} N\left(0, E \frac{I(Y>t) \delta}{S_{C}^{2}(Y-A)}-M^{2}(t)\right),
\end{aligned}
$$

where $\stackrel{\mathscr{L}}{\longrightarrow}$ denotes weak convergence.

Since

$$
\begin{aligned}
&\left|\frac{1}{n} \sum_{i=1}^{n} \frac{I\left(y_{i}>t\right) \delta_{i}}{\widehat{S}_{C}\left(y_{i}-a_{i}\right)}-M(t)\right| \\
&=\mid \frac{1}{n} \sum_{i=1}^{n} \frac{I\left(y_{i}>t\right) \delta_{i}}{\widehat{S}_{C}\left(y_{i}-a_{i}\right)}-\frac{1}{n} \sum_{i=1}^{n} \frac{I\left(y_{i}>t\right) \delta_{i}}{S_{C}\left(y_{i}-a_{i}\right)} \\
& \quad+\frac{1}{n} \sum_{i=1}^{n} \frac{I\left(y_{i}>t\right) \delta_{i}}{S_{C}\left(y_{i}-a_{i}\right)}-M(t) \mid \\
& \leq\left|\frac{1}{n} \sum_{i=1}^{n} \frac{I\left(y_{i}>t\right) \delta_{i}}{\widehat{S}_{C}\left(y_{i}-a_{i}\right)}-\frac{1}{n} \sum_{i=1}^{n} \frac{I\left(y_{i}>t\right) \delta_{i}}{S_{C}\left(y_{i}-a_{i}\right)}\right| \\
& \quad+\left|\frac{1}{n} \sum_{i=1}^{n} \frac{I\left(y_{i}>t\right) \delta_{i}}{S_{C}\left(y_{i}-a_{i}\right)}-M(t)\right| \\
& \leq \frac{1}{n} \sum_{i=1}^{n} I\left(y_{i}>t\right) \delta_{i}\left|\frac{1}{\widehat{S}_{C}\left(y_{i}-a_{i}\right)}-\frac{1}{S_{C}\left(y_{i}-a_{i}\right)}\right| \\
&+\left|\frac{1}{n} \sum_{i=1}^{n} \frac{I\left(y_{i}>t\right) \delta_{i}}{S_{C}\left(y_{i}-a_{i}\right)}-M(t)\right|,
\end{aligned}
$$


and recall that $\widehat{S}_{C}(\cdot)$ is the product-limit estimator of $S_{C}(\cdot)$, so on the basis of (14) and the consistency of $\widehat{S}_{C}(\cdot)$ (see [14]), we can obtain

$$
\begin{gathered}
\left|\frac{1}{n} \sum_{i=1}^{n} \frac{I\left(y_{i}>t\right) \delta_{i}}{\widehat{S}_{C}\left(y_{i}-a_{i}\right)}-M(t)\right| \longrightarrow 0, \quad n \rightarrow \infty, \\
\operatorname{Var}\left(Z_{1 n}\right)=E \frac{I\left(y_{i}>t\right) \delta_{i}}{\widehat{S}_{C}^{2}\left(y_{i}-a_{i}\right)}-M^{2}(t) \\
\longrightarrow E \frac{I(Y>t) \delta}{S_{C}^{2}(Y-A)}-M^{2}(t), \quad n \longrightarrow \infty .
\end{gathered}
$$

Since $I\left(y_{i}>t\right) \delta_{i} / \widehat{S}_{C}\left(y_{i}-a_{i}\right), i=1,2, \ldots, n$, are i.i.d, it is easy to derive the result of Lemma 2(i) by the central limit theorem.

Using the same method, let us give the proof of Lemma 2 (ii). Under the regular conditions (i-iv) and (12), because $E\left(I(Y>t) \delta / Y S_{C}(Y-A)\right)=N(t)$, by the central limit theorem, we can derive

$$
\begin{aligned}
& \sqrt{n}\left(\frac{1}{n} \sum_{i=1}^{n} \frac{I\left(y_{i}>t\right) \delta_{i}}{y_{i} S_{C}\left(y_{i}-a_{i}\right)}-N(t)\right) \\
& \stackrel{\mathscr{L}}{\longrightarrow} N\left(0, E \frac{I(Y>t) \delta}{Y^{2} S_{C}^{2}(Y-A)}-N^{2}(t)\right) .
\end{aligned}
$$

Since

$$
\begin{aligned}
&\left|\frac{1}{n} \sum_{i=1}^{n} \frac{I\left(y_{i}>t\right) \delta_{i}}{y_{i} \widehat{S}_{C}\left(y_{i}-a_{i}\right)}-N(t)\right| \\
&=\mid \frac{1}{n} \sum_{i=1}^{n} \frac{I\left(y_{i}>t\right) \delta_{i}}{y_{i} \widehat{S}_{C}\left(y_{i}-a_{i}\right)}-\frac{1}{n} \sum_{i=1}^{n} \frac{I\left(y_{i}>t\right) \delta_{i}}{y_{i} S_{C}\left(y_{i}-a_{i}\right)} \\
& \quad+\frac{1}{n} \sum_{i=1}^{n} \frac{I\left(y_{i}>t\right) \delta_{i}}{y_{i} S_{C}\left(y_{i}-a_{i}\right)}-N(t) \mid \\
& \leq\left|\frac{1}{n} \sum_{i=1}^{n} \frac{I\left(y_{i}>t\right) \delta_{i}}{y_{i} \widehat{S}_{C}\left(y_{i}-a_{i}\right)}-\frac{1}{n} \sum_{i=1}^{n} \frac{I\left(y_{i}>t\right) \delta_{i}}{y_{i}\left(y_{i}-a_{i}\right)}\right| \\
& \quad+\left|\frac{1}{n} \sum_{i=1}^{n} \frac{I\left(y_{i}>t\right) \delta_{i}}{y_{i} S_{C}\left(y_{i}-a_{i}\right)}-N(t)\right| \\
& \leq \frac{1}{n} \sum_{i=1}^{n} I\left(y_{i}>t\right) \delta_{i} y_{i}^{-1}\left|\frac{1}{\widehat{S}_{C}\left(y_{i}-a_{i}\right)}-\frac{1}{S_{C}\left(y_{i}-a_{i}\right)}\right| \\
&+\left|\frac{1}{n} \sum_{i=1}^{n} \frac{I\left(y_{i}>t\right) \delta_{i}}{y_{i} S_{C}\left(y_{i}-a_{i}\right)}-N(t)\right|
\end{aligned}
$$

so on the basis of (17) and the consistency of $\widehat{S}_{C}(\cdot)$, we can derive

$$
\begin{aligned}
\left|\frac{1}{n} \sum_{i=1}^{n} \frac{I\left(y_{i}>t\right) \delta_{i}}{y_{i} \widehat{S}_{C}\left(y_{i}-a_{i}\right)}-N(t)\right| \rightarrow 0, \quad n \rightarrow \infty, \\
\operatorname{Var}\left(Z_{2 n}\right)=E \frac{I\left(y_{i}>t\right) \delta_{i}}{y_{i}^{2} \widehat{S}_{C}^{2}\left(y_{i}-a_{i}\right)}-N^{2}(t) \\
\longrightarrow E \frac{I(Y>t) \delta}{Y^{2} S_{C}^{2}(Y-A)}-N^{2}(t), \quad n \longrightarrow \infty .
\end{aligned}
$$

Since $I\left(y_{i}>t\right) \delta_{i} / y_{i} \widehat{S}_{C}\left(y_{i}-a_{i}\right), i=1,2, \ldots, n$, are i.i.d, it is easy to derive the result of Lemma 2(ii) by the central limit theorem. The proof of Lemma 2 is completed.

On the basis of Lemmas 1 and 2, now we can discuss the asymptotic property of $\widetilde{m}(t)$.

Theorem 3. Assume the conditions (i-iv) and (12) hold, for every $t \in[0, L]$, as $n \rightarrow \infty$, then $\sqrt{n}(\widetilde{m}(t)-m(t))$ converges in distribution to a zero mean normal variable with the variance function $\left(E Z_{1}^{2}(t) / N^{2}(t)\right)-\left(M(t) E Z_{1}(t) Z_{2}(t) / N^{3}(t)\right)+$ $\left(M^{2}(t) E Z_{2}^{2}(t) / N^{4}(t)\right)$.

Proof. Under the conditions (i-iv) and (12), Lemma 2 allows a straightforward application of Lemma 1 . So we can derive

$$
\sqrt{n}(\widetilde{m}(t)-m(t)) \stackrel{d}{\longrightarrow} \mathcal{N}_{\widetilde{m}}=\frac{Z_{1}(t)}{N(t)}-\frac{M(t) Z_{2}(t)}{N^{2}(t)},
$$

where $\stackrel{d}{\rightarrow}$ denotes convergence in distribution. $\mathcal{N}_{\widetilde{m}}$ is Gaussian as a linear form on two Gaussian variables and it is easy to check that $E \mathcal{N}_{\widetilde{m}}=0$, since $Z_{1}$ and $Z_{2}$ are centered. It is also easy to calculate the variance of $\mathcal{N}_{\widetilde{m}}$ and it can be expressed as

$$
\begin{aligned}
\operatorname{Var}\left(\mathcal{N}_{\widetilde{m}}\right)= & \frac{E Z_{1}^{2}(t)}{N^{2}(t)}-\frac{M(t) E Z_{1}(t) Z_{2}(t)}{N^{3}(t)} \\
& +\frac{M^{2}(t) E Z_{2}^{2}(t)}{N^{4}(t)} .
\end{aligned}
$$

The proof is completed.

\section{Simulations}

To compare the performance of our proposed estimator with the one in (6), we conducted a series of Monte Carlo simulations with $n=100$ and 200. In these simulations, the underlying survival time $T^{0}$ has a uniform $(0,1)$ distribution. Then the length-biased density function of $T$ is $g(t)=$ $2 t, t \in(0,1)$, and the left-truncated time $A$ is uniformly distributed on $[0, T]$. We simulated the censoring time $C$ from either a uniform $(0,1)$, a uniform $(0,5 / 3)$ or a uniform $(0,5)$ distribution, corresponding to a censoring rate of $50 \%, 30 \%$, and $10 \%$.

Table 1 summarizes the Monte Carlo bias, standard deviation, and mean square error for each estimator 
TABLE 1: Simulation results for nonparametric estimators based on 1000 replications.

\begin{tabular}{|c|c|c|c|c|c|c|c|}
\hline \multirow{2}{*}{$t$} & \multirow{2}{*}{$m(t)$} & \multicolumn{3}{|c|}{$\widehat{m}(t)$} & \multicolumn{3}{|c|}{$\widetilde{m}(t)$} \\
\hline & & Bias & SE & MSE & Bias & SE & MSE \\
\hline \multicolumn{8}{|c|}{$n=100, C \sim \operatorname{Un}(0,5)$} \\
\hline 0.2 & 0.4 & 51 & 28 & 34 & 2 & 29 & 8 \\
\hline 0.4 & 0.3 & 50 & 21 & 30 & 0 & 20 & 4 \\
\hline 0.6 & 0.2 & 50 & 16 & 28 & 0 & 15 & 2 \\
\hline 0.8 & 0.1 & 50 & 14 & 27 & 0 & 11 & 1 \\
\hline \multicolumn{8}{|c|}{$n=200, C \sim \operatorname{Un}(0,5)$} \\
\hline 0.2 & 0.4 & 51 & 20 & 30 & 1 & 20 & 4 \\
\hline 0.4 & 0.3 & 51 & 14 & 28 & 1 & 14 & 2 \\
\hline 0.6 & 0.2 & 50 & 11 & 26 & 0 & 11 & 1 \\
\hline 0.8 & 0.1 & 50 & 7 & 25 & 0 & 7 & 1 \\
\hline \multicolumn{8}{|c|}{$n=100, C \sim \operatorname{Un}(0,5 / 3)$} \\
\hline 0.2 & 0.4 & 49 & 32 & 35 & 1 & 30 & 9 \\
\hline 0.4 & 0.3 & 49 & 28 & 31 & 1 & 22 & 5 \\
\hline 0.6 & 0.2 & 48 & 29 & 31 & 0 & 17 & 3 \\
\hline 0.8 & 0.1 & 45 & 56 & 51 & 0 & 12 & 1 \\
\hline \multicolumn{8}{|c|}{$n=200, C \sim \operatorname{Un}(0,5 / 3)$} \\
\hline 0.2 & 0.4 & 50 & 21 & 30 & 1 & 21 & 5 \\
\hline 0.4 & 0.3 & 50 & 16 & 28 & 0 & 15 & 2 \\
\hline 0.6 & 0.2 & 50 & 15 & 28 & 1 & 12 & 1 \\
\hline 0.8 & 0.1 & 51 & 15 & 28 & 1 & 8 & 1 \\
\hline \multicolumn{8}{|c|}{$n=100, C \sim \operatorname{Un}(0,1)$} \\
\hline 0.2 & 0.4 & 49 & 62 & 62 & 2 & 32 & 10 \\
\hline 0.4 & 0.3 & 53 & 233 & 572 & -2 & 26 & 7 \\
\hline 0.6 & 0.2 & 37 & 160 & 268 & 5 & 21 & 5 \\
\hline 0.8 & 0.1 & 107 & 1733 & 30130 & -1 & 15 & 2 \\
\hline \multicolumn{8}{|c|}{$n=200, C \sim \operatorname{Un}(0,1)$} \\
\hline 0.2 & 0.4 & 49 & 24 & 30 & 2 & 22 & 5 \\
\hline 0.4 & 0.3 & 50 & 43 & 43 & 2 & 19 & 4 \\
\hline 0.6 & 0.2 & 48 & 26 & 30 & -2 & 14 & 2 \\
\hline 0.8 & 0.1 & 53 & 134 & 208 & -1 & 11 & 1 \\
\hline
\end{tabular}

Notes for the numerical results based on 1000 replications in Table 1:

(1) Bias $=$ the empirical bias $\times 10^{3}$;

(2) $\mathrm{SE}=$ the empirical standard deviation $\times 10^{3}$;

(3) MSE $=$ the empirical mean square error $\times 10^{4}$.

at $t=0.2,0.4,0.6$ and 0.8 based on 1000 replications. The survival probabilities at the selected time-points are $0.8,0.6$, 0.4 , and 0.2 . The corresponding true values of $m(t)$ at the selected time-points are $0.4,0.3,0.2$, and 0.1 , respectively.

The outcomes show that the proposed estimator works very well. Through the numerical results in Table 1, we derive the following conclusions:

(1) $\widetilde{m}(t)$ outperforms $\widehat{m}(t)$ obviously in terms of Monte Carlo bias and mean square error for every values of $t$ when $n=200$ or $n=100$;

(2) the two estimators are very close to each other in terms of the Monte Carlo standard deviation when the censoring rate is small;
(3) when the censoring rate is heavy, $\widetilde{m}(t)$ outperforms $\widehat{m}(t)$ obviously in all terms that we referred to in the simulations.

Through the conclusions given above, it is appropriate to explore our proposed method in real applications.

\section{Conflict of Interests}

The authors declare that there is no conflict of interests regarding the publication of this paper.

\section{Acknowledgment}

This research is supported by the National Science Foundation of China Grants 11371227 and 10921101.

\section{References}

[1] M. Csörgö and R. Zitikis, "Meant residual life processes," The Annals of Statistics, vol. 24, no. 4, pp. 1717-1739, 1996.

[2] G. L. Yang, "Estimation of a biometric function," The Annals of Statistics, vol. 6, no. 1, pp. 112-116, 1978.

[3] G. Yang, "Life expectancy under random censorship," Stochastic Processes and Their Applications, vol. 6, no. 1, pp. 33-39, 1977.

[4] Y. Kumazawa, "A note on an estimator of life expectancy with random censorship," Biometrika, vol. 74, no. 3, pp. 655-658, 1987.

[5] Y. C. Zhao and G. S. Qin, "Inference for the mean residual life function via empirical likelihood," Communications in Statistics-Theory and Methods, vol. 35, no. 6, pp. 1025-1036, 2006.

[6] L. Q. Sun and Z. G. Zhang, "A class of transformed mean residual life models with censored survival data," Journal of the American Statistical Association, vol. 104, no. 486, pp. 803-815, 2009.

[7] M. Zhao, H. M. Jiang, and X. Liu, "A note on estimation of the mean residual life function with left-truncated and rightcensored data," Statistics \& Probability Letters, vol. 83, no. 10, pp. 2332-2336, 2013.

[8] C. Y. Huang and J. Qin, "Nonparametric estimation for lengthbiased and right-censored data," Biometrika, vol. 98, no. 1, pp. 177-186, 2011.

[9] Y. Vardi, "Nonparametric estimation in the presence of length bias," The Annals of Statistics, vol. 10, pp. 616-620, 1982.

[10] R. C. Gupta and J. P. Keating, "Relations for reliability measures under length-biased sampling," Scandinavian Journal of Statistics, vol. 13, pp. 49-56, 1986.

[11] P. J. Bergeron, M. Asgharian, and D. B. Wolfson, "Covariate bias induced by length-biased sampling of failure times," Journal of the American Statistical Association, vol. 103, no. 482, pp. 737$742,2008$.

[12] X. D. Luo and W. Y. Tsai, "Nonparametric estimation for right-censored length-biased data: a pseudo-partial likelihood approach," Biometrika, vol. 96, no. 4, pp. 873-886, 2009.

[13] T. Lancaster, The Econonetric Analysis of Transition Data, Cambridge University Press, Cambridge, UK, 1990.

[14] B. N. Breslow and J. Crowley, "A large sample study of the life table and product limit estimates under random censorship," The Annals of Statistics, vol. 2, no. 3, pp. 437-453, 1974. 


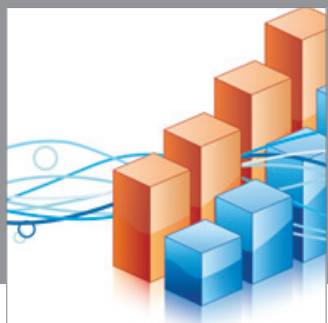

Advances in

Operations Research

mansans

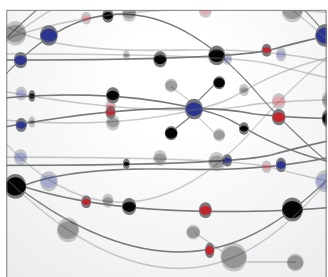

The Scientific World Journal
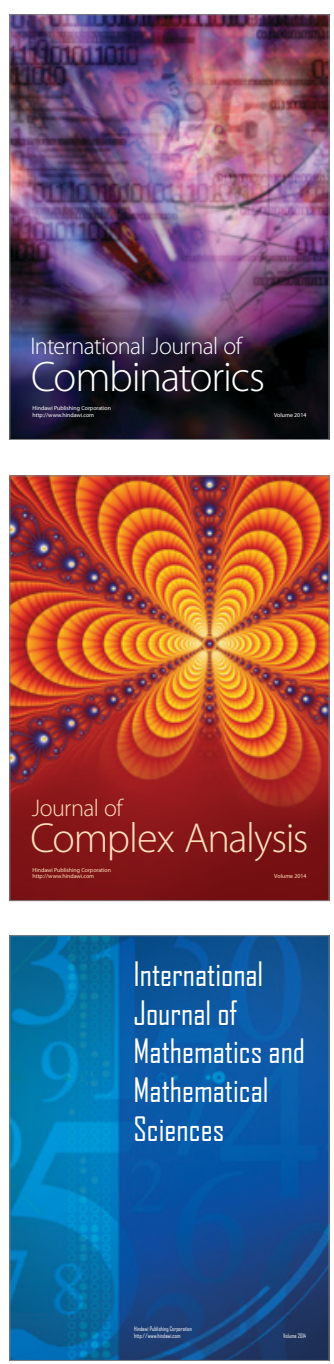
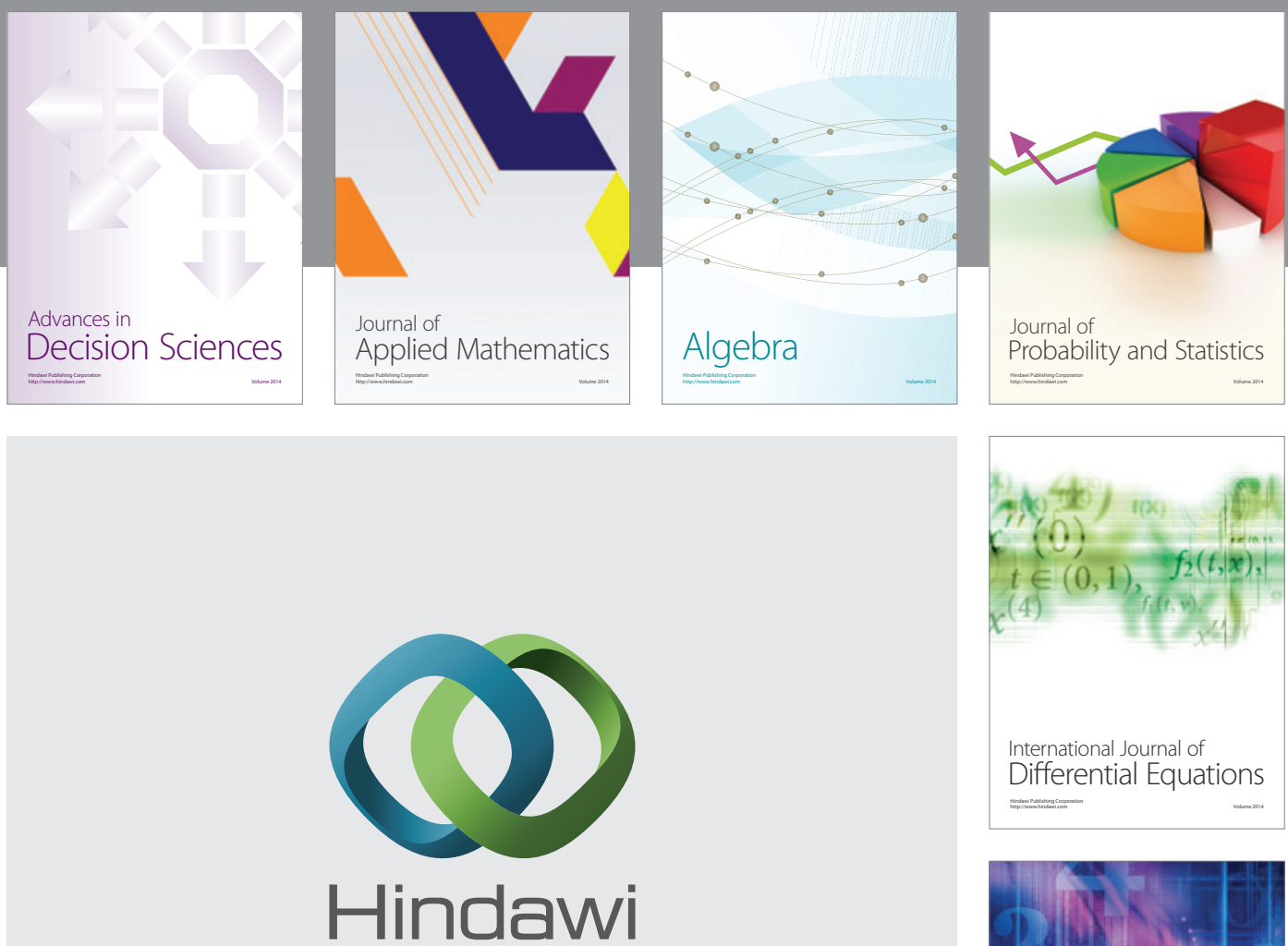

Submit your manuscripts at http://www.hindawi.com
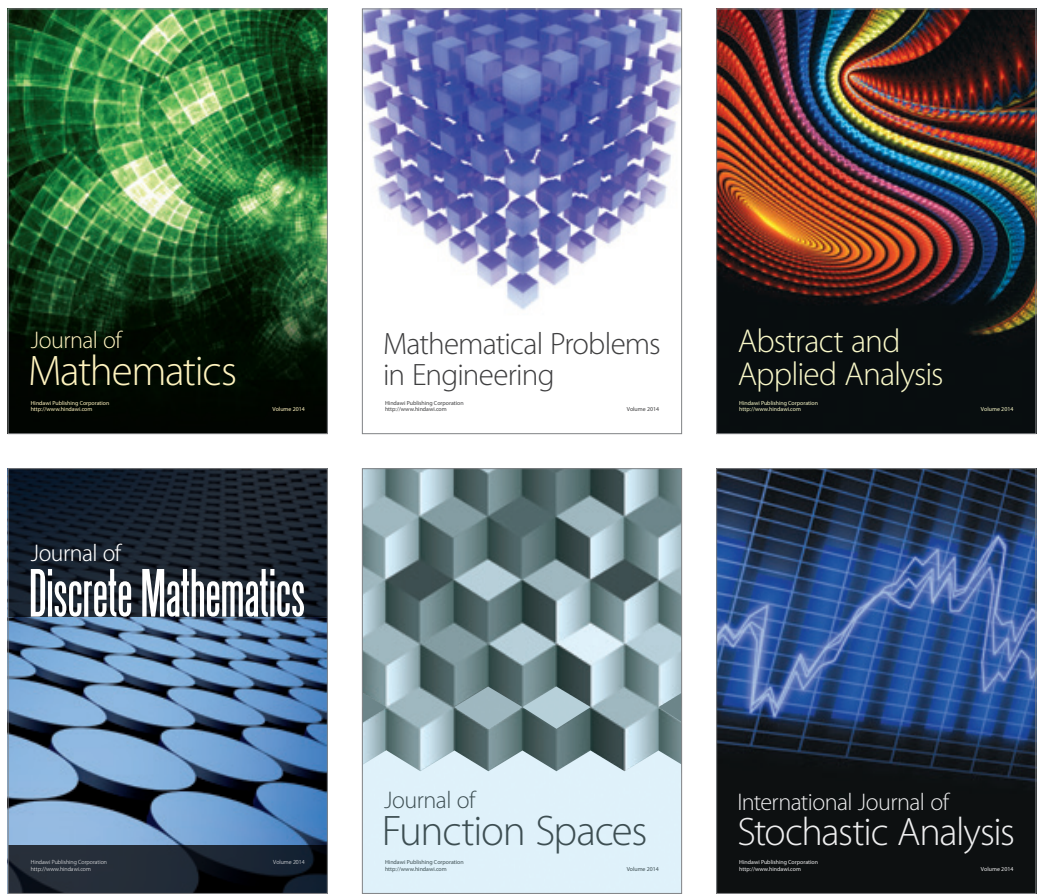

Journal of

Function Spaces

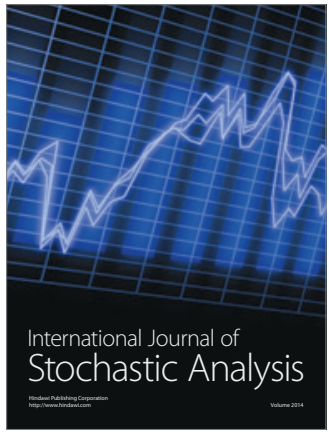

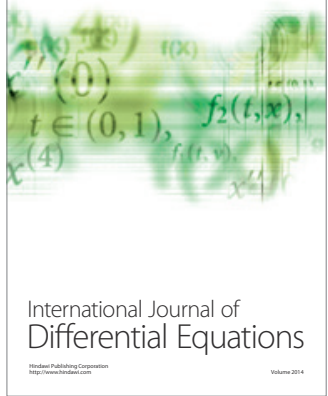
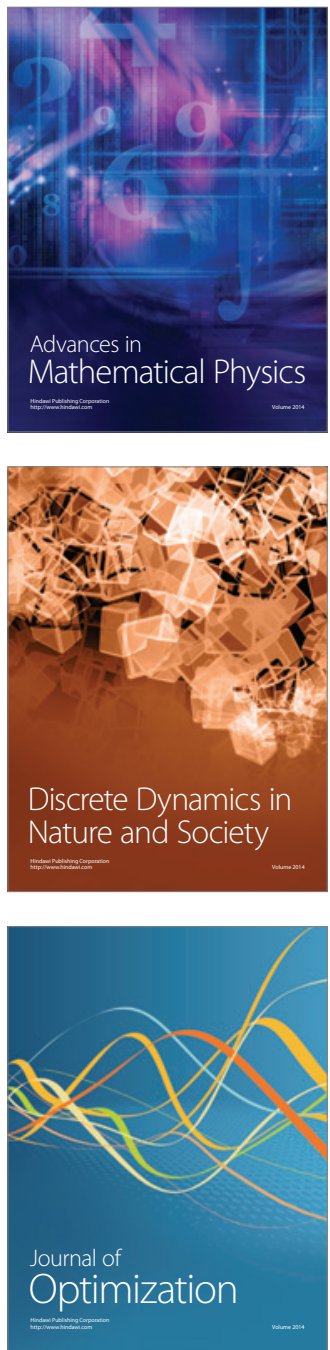